\title{
A two-input fluorescent probe for thiols and hydrogen sulfide chemosensing and live cell imaging
}

\author{
Chun-Guang Dai, ${ }^{[\mathrm{a}]}$ Xiu-Ling Liu, ${ }^{[\mathrm{a}]}$ Xiao-Jiao Du, ${ }^{[\mathrm{b}]}$ Yan Zhang $^{[\mathrm{c}]}$ and Qin-Hua Song* ${ }^{[\mathrm{a}]}$ \\ ${ }^{a}$ Department of Chemistry, University of Science and Technology of China, Hefei 230026, P. R. China. ${ }^{b}$ School \\ of Life Sciences, University of Science and Technology of China, Hefei 230027, P. R. China. ${ }^{\mathrm{c}}$ State Key \\ Laboratory of Analytical Chemistry for Life Science, Institute of Chemistry \& BioMedical Sciences, School of \\ Chemistry and Chemical Engineering, Nanjing University, Nanjing, 210093, P. R. China. \\ E-mail:qhsong@ustc.edu.cn
}

\section{Contents}

I. UV/vis absorption and fluorescence spectra of related compounds...................S2

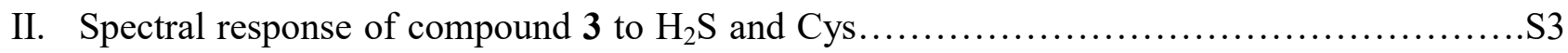

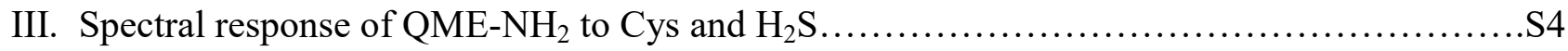

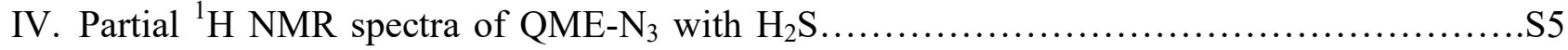

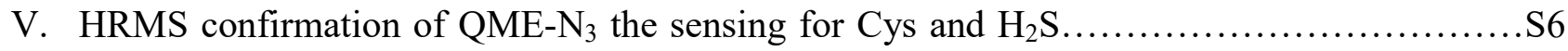

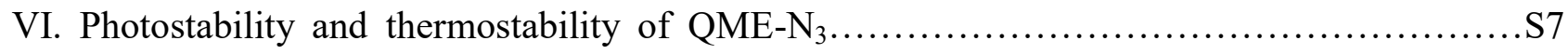

VII. Synthesis and characterization data of related compounds........................... 8 -11

VIII. Copies of NMR spectra of related compounds................................. $12-17$ 
I. UV/vis absorption and fluorescence spectra of compounds 2, 3, QME-N $\mathrm{N}_{3}$ and QME- $\mathrm{NH}_{2}$ and QME-NH${ }_{2}-\mathrm{Pr}$
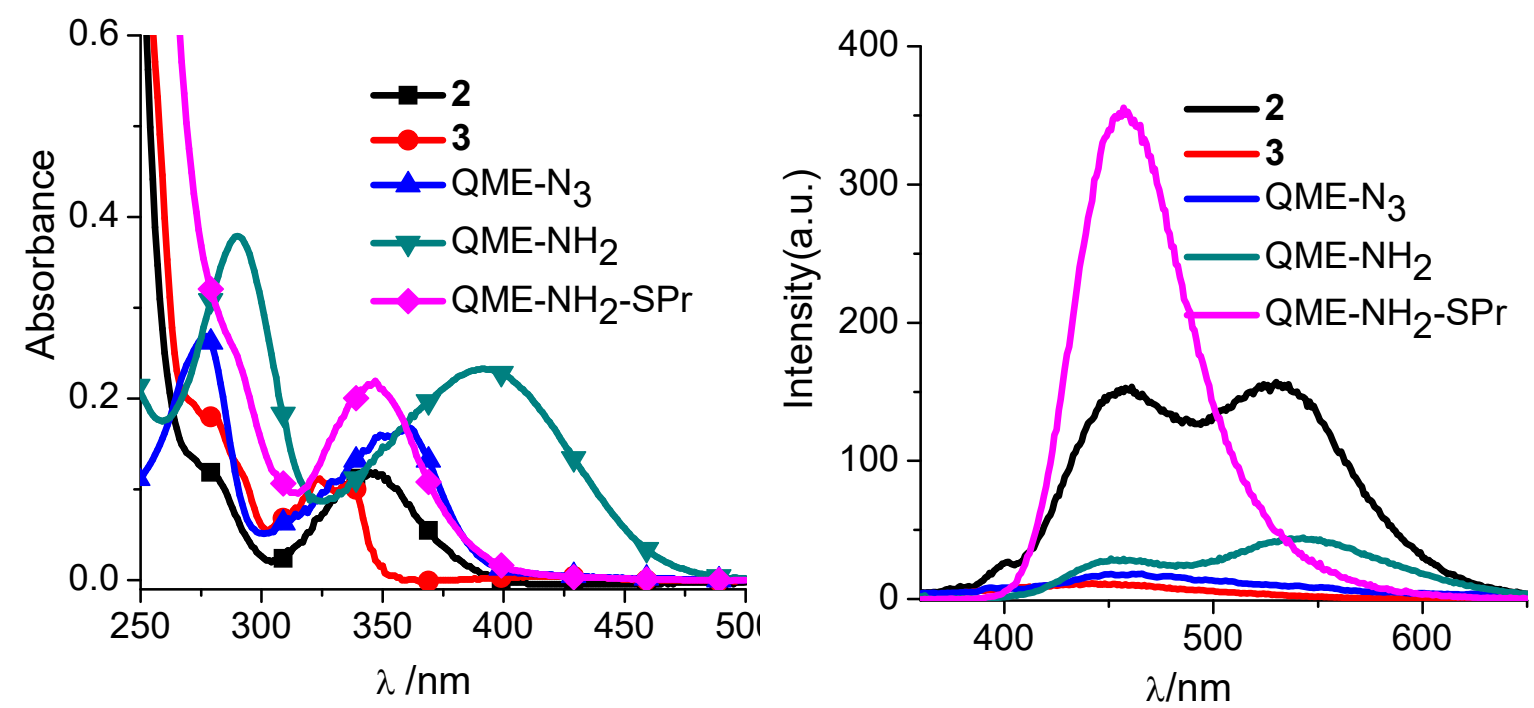

Figure S1. UV/vis absorption (left) and fluorescence spectra (right) of $20 \mu \mathrm{M}$ compounds 2, 3, QME-N 3, QME-NH ${ }_{2}$ and QME-NH ${ }_{2}-\mathrm{SPr}$ in the PBS-ethanol (v/v, 3:1, $\left.\mathrm{pH} 7.4\right)$ solutions. $\lambda_{\text {ex }}=350$ nm. 
II. Spectral response of compound 3 to $\mathrm{H}_{2} \mathrm{~S}$ and Cys
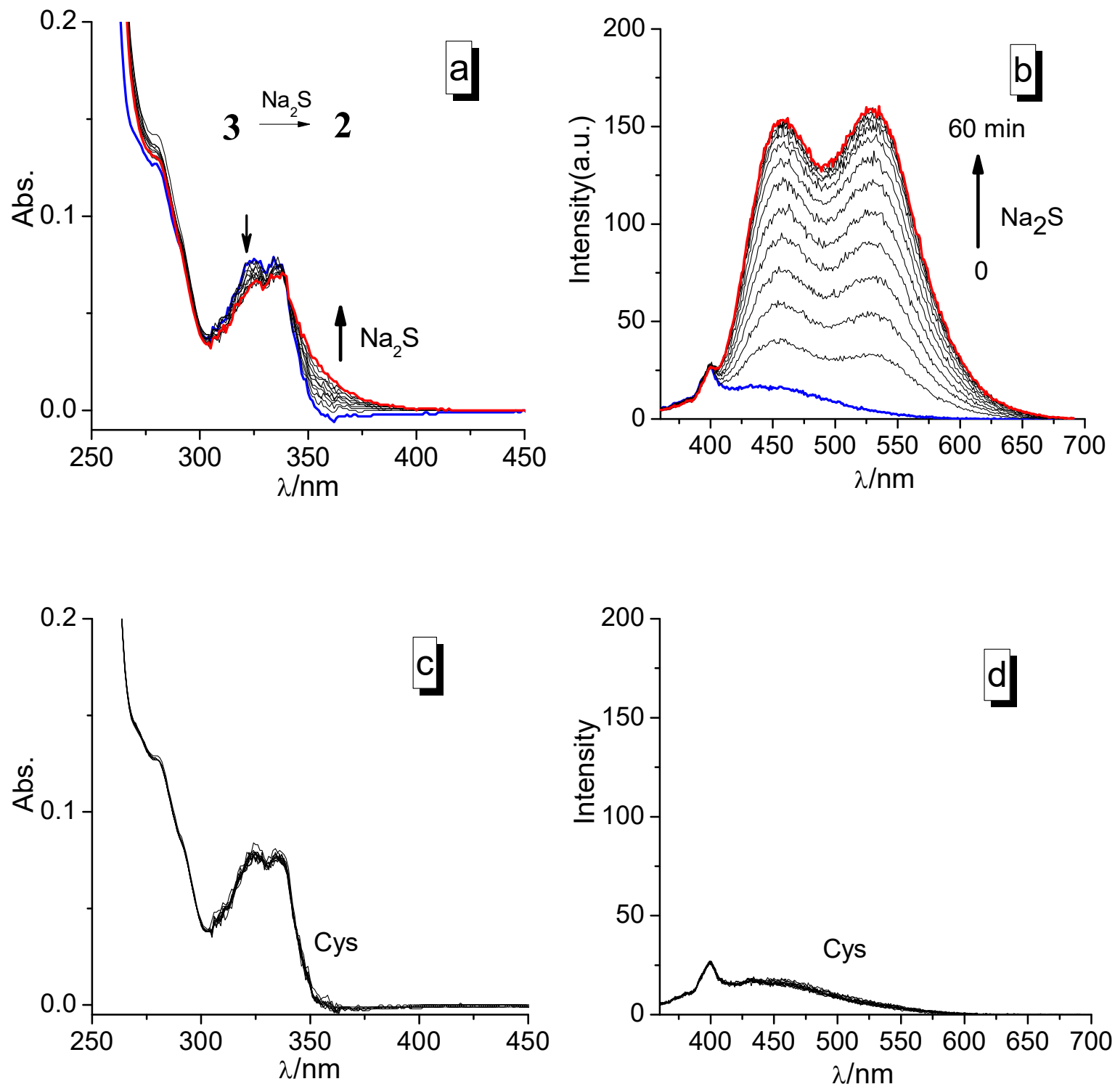

Figure S2. Time-dependent UV/vis absorption (a, c) and fluorescence spectra (b, d) of compound 3 $(20 \mu \mathrm{M})$ in the presence of 100 equiv. $\mathrm{Na}_{2} \mathrm{~S}(\mathrm{a}, \mathrm{b})$ or 10 equiv. Cys $(\mathrm{c}, \mathrm{d})$ for $60 \mathrm{~min}$ in PBS (pH 7.4) buffered water-ethanol (v/v, 3:1). $\lambda_{\mathrm{ex}}=350 \mathrm{~nm}$. 
III. Spectral response of QME- $\mathrm{NH}_{2}$ to Cys and $\mathrm{H}_{2} \mathrm{~S}$
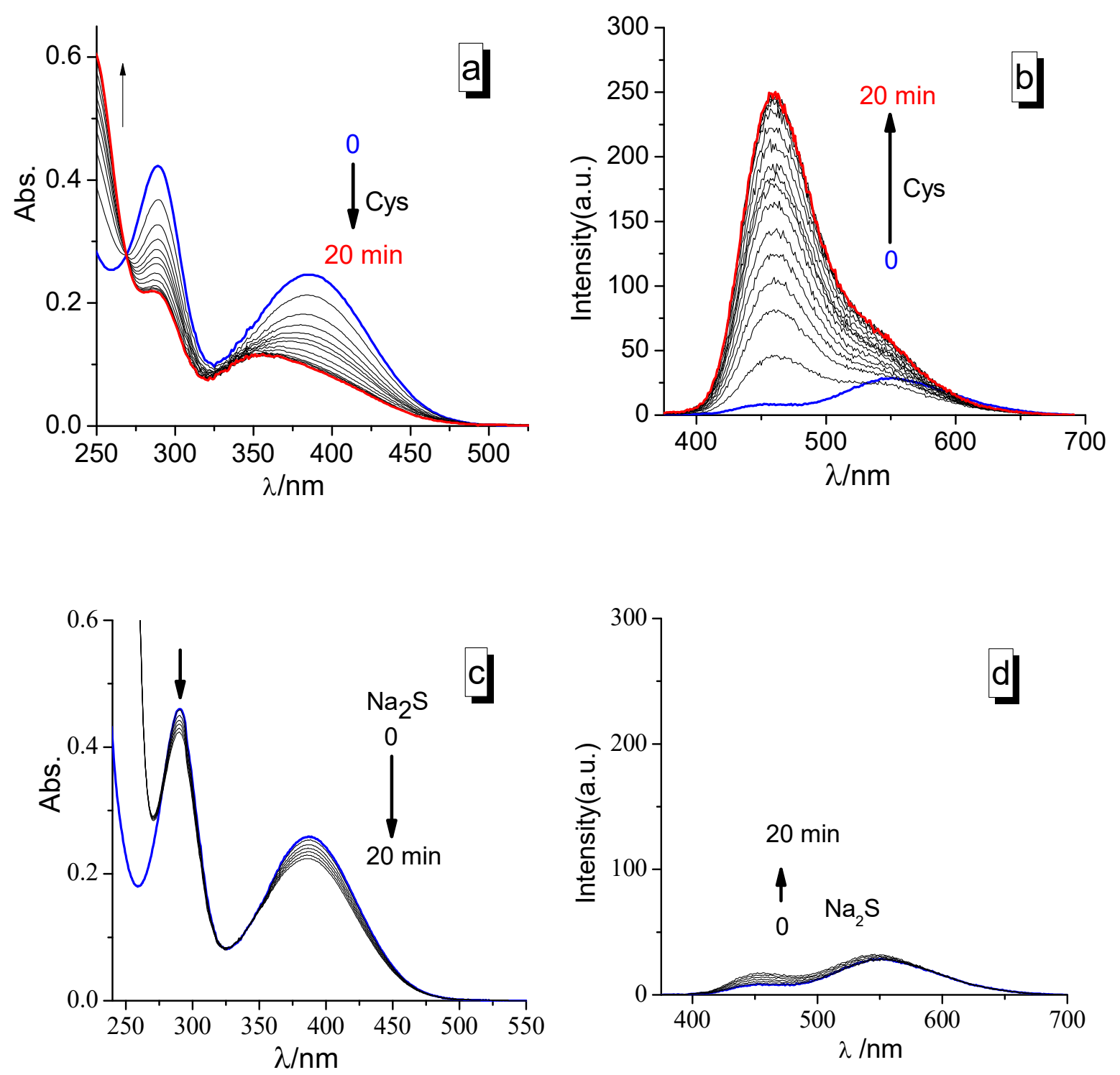

Figure S3. Time-dependent Uv/vis absorption (a, c) and fluorescence spectra (b, d) of QME- $\mathrm{NH}_{2}$ $(20 \mu \mathrm{M})$ in the presence of 10 equiv. Cys $(\mathrm{a}, \mathrm{b})$ for $20 \mathrm{~min}$ or 100 equiv. $\mathrm{Na}_{2} \mathrm{~S}(\mathrm{c}, \mathrm{d})$ for $60 \mathrm{~min}$ in PBS (pH 7.4) buffered water-ethanol (v/v, 3:1). $\lambda_{\mathrm{ex}}=320 \mathrm{~nm}$. 
IV. Partial ${ }^{1} \mathrm{H}$ NMR Spectra of QME- $\mathrm{N}_{3}$ with $\mathrm{Na}_{2} \mathrm{~S}$
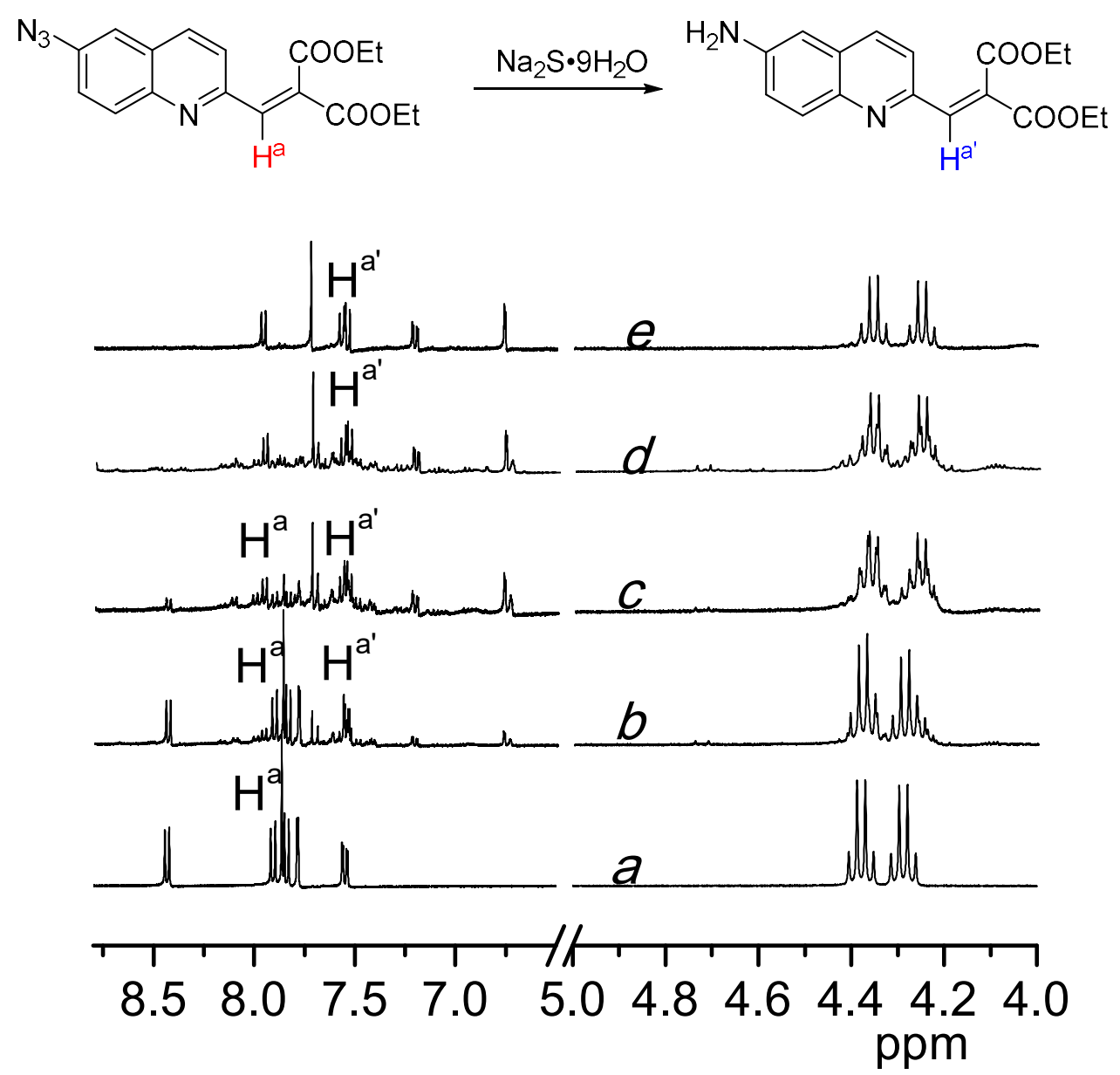

Figure S4. Partial ${ }^{1} \mathrm{H}$ NMR spectra of QME-N $\mathrm{N}_{3}$ (a), QME-N $\mathrm{N}_{3}$ with $\mathrm{Na}_{2} \mathrm{~S} \cdot 9 \mathrm{H}_{2} \mathrm{O}$ (b, c, d) recorded at three time intervals and QME- $\mathrm{NH}_{2}(\mathrm{e})$ in DMSO- $d_{6}$. 
V. HRMS confirmation of QME- $\mathrm{N}_{3}$ the sensing for Cys and $\mathrm{H}_{2} \mathrm{~S}$
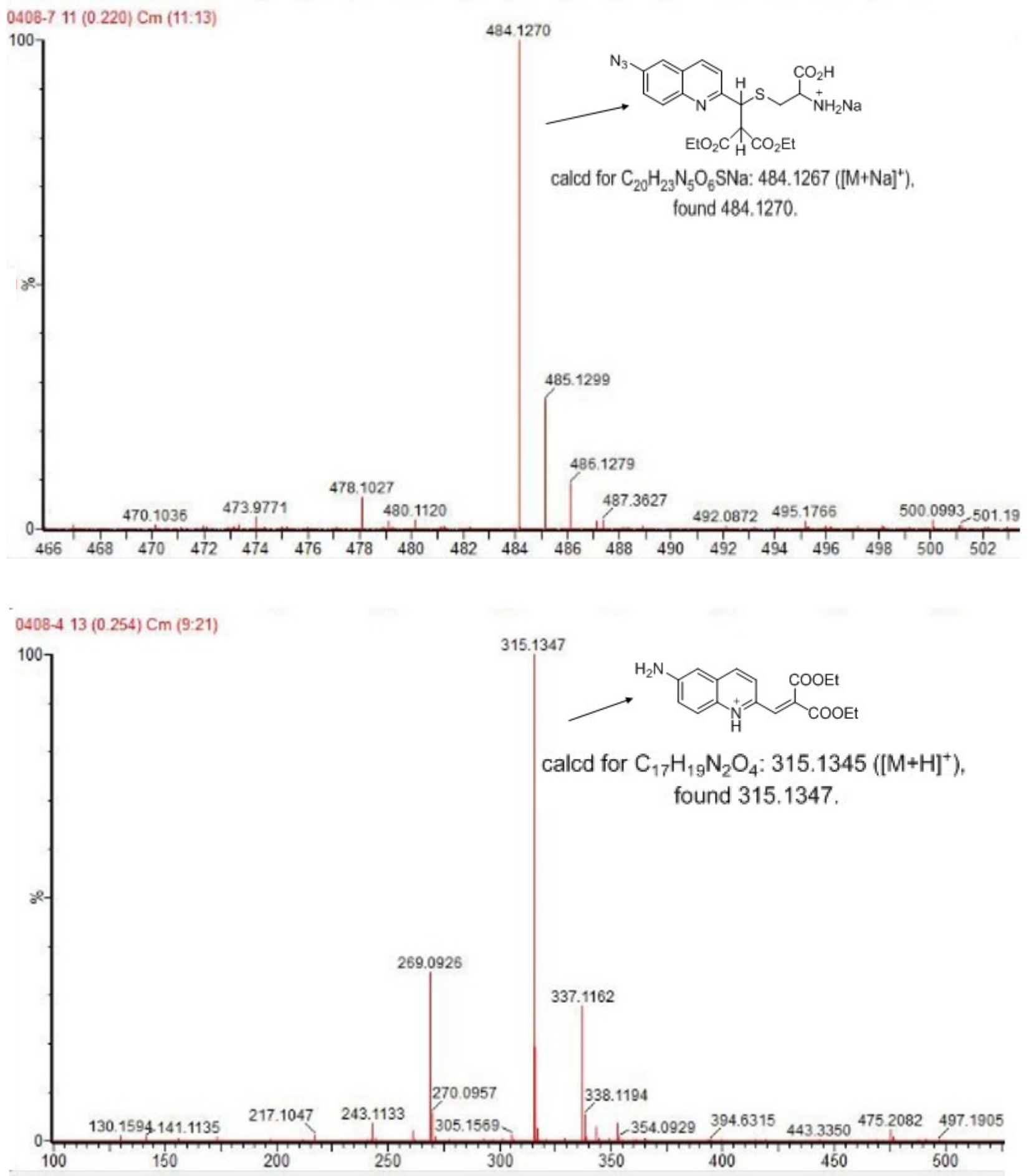

Figure S5. Mass spectra of the reaction mixture of QME- $\mathrm{N}_{3}$ incubated with Cys (up) or $\mathrm{H}_{2} \mathrm{~S}$ (down) in PBS (pH 7.4) buffered water-ethanol (v/v, 3:1). 


\section{Photostability of QME-N 3}

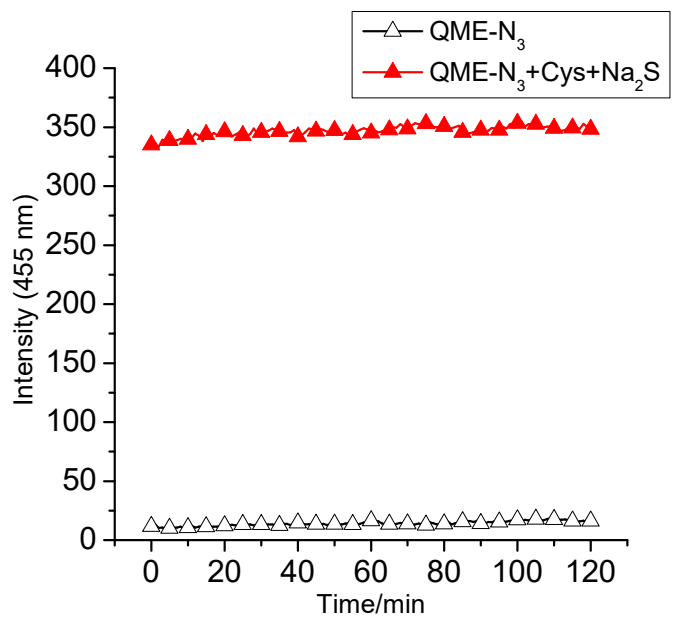

Figure S6. Fluorescence photoactivation response of $\mathrm{QME}^{-\mathrm{N}_{3}}(20 \mu \mathrm{M})$ responding to Cys (10 equiv.) and $\mathrm{Na}_{2} \mathrm{~S}$ (100 equiv.) in aqueous solution (ethanol/PBS, v/v 1:3, pH 7.4) respectively under continuous irradiation by a xenon lamp $(150 \mathrm{~W})$ within $120 \mathrm{~min}, \lambda_{\mathrm{ex}}=320 \mathrm{~nm}, \lambda_{\mathrm{em}}=455 \mathrm{~nm}$ Excitation slits: 3,3 . Data measured at $1 \mathrm{~min}^{-1}$.
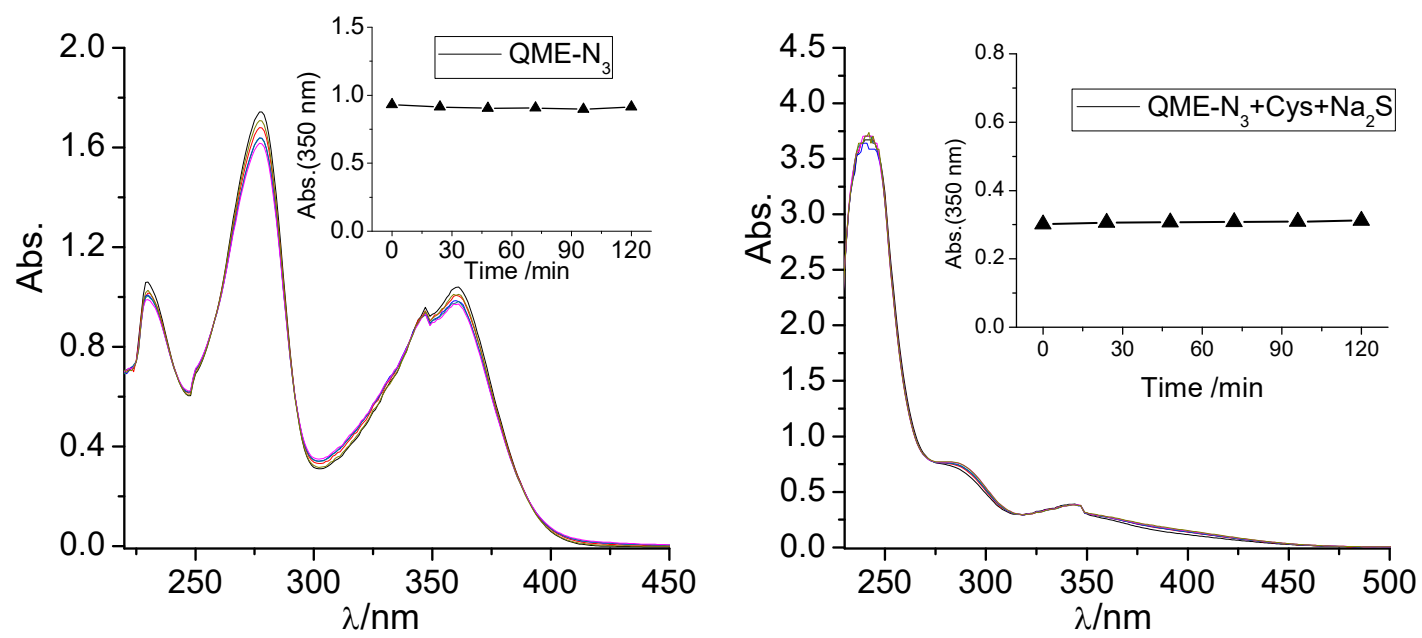

Figure S7. The time courses of Uv/vis spectra of QME-N $\mathrm{N}_{3}(20 \mu \mathrm{M})$ responding to Cys (10 equiv.) and $\mathrm{Na}_{2} \mathrm{~S}$ (100 equiv.) in aqueous solution (ethanol/PBS, v/v 1:3, pH 7.4) in $120 \mathrm{~min}$. 


\section{Synthesis and Characterization Data of Related Compounds}

2-Methyl-6-nitroquinoline (1). ${ }^{1}{ }^{1} \mathrm{H}$ NMR (400 MHz, $\left.\mathrm{CDCl}_{3}, \mathrm{TMS}\right): \delta=8.75(\mathrm{~d}, J=2.5 \mathrm{~Hz}, 1 \mathrm{H})$, $8.45\left(\mathrm{dd}, J_{l}=2.5, J_{2}=9.2 \mathrm{~Hz}, 1 \mathrm{H}\right), 8.23(\mathrm{~d}, J=8.5 \mathrm{~Hz}, 1 \mathrm{H}), 8.13(\mathrm{~d}, J=9.2 \mathrm{~Hz}, 1 \mathrm{H}), 7.46(\mathrm{~d}, J=$ $8.5 \mathrm{~Hz}, 1 \mathrm{H}), 2.81\left(\mathrm{~s}, 3 \mathrm{H}, \mathrm{CH}_{3}\right)$.

2-Methylquinolin-6-amine (2). ${ }^{1} \mathrm{H}$ NMR (400 MHz, $\mathrm{CDCl}_{3}$, TMS): $\delta=7.81(\mathrm{~m}, 2 \mathrm{H}), 7.16(\mathrm{~d}, J=$ $8.3 \mathrm{~Hz}, 1 \mathrm{H}), 7.13\left(\mathrm{dd}, J_{1}=8.8, J_{2}=2.6 \mathrm{~Hz}, 1 \mathrm{H}\right), 6.88(\mathrm{~d}, J=2.5 \mathrm{~Hz}, 1 \mathrm{H}), 3.88\left(\mathrm{br}, 2 \mathrm{H}, \mathrm{NH}_{2}\right), 2.67$ $\left(\mathrm{s}, 3 \mathrm{H}, \mathrm{CH}_{3}\right)$.

6-Azido-2-methylquinoline (3). To a $100 \mathrm{~mL}$ round bottom flask equipped with a magnetic stir bar, 2-methylquinolin-6-amine (1.0 g, $6.32 \mathrm{mmol})$ was added followed by adding $\mathrm{HCl}(6 \mathrm{M}, 24 \mathrm{~mL})$ at $0{ }^{\circ} \mathrm{C}$. The mixture was stirred for $1 \mathrm{~h}$, then $\mathrm{NaNO}_{2}(4.36 \mathrm{~g}, 63.2 \mathrm{mmol})$ was added into the solution in portions. Then the mixture was stirred for more another hour after $\mathrm{NaN}_{3}(4.11 \mathrm{~g}, 63.2 \mathrm{mmol})$ was added portion wise. The mixture was extracted with ether $(3 \times 25 \mathrm{~mL})$ and then the ether extracts were washed with distilled water $(2 \times 50 \mathrm{~mL})$ and brine $(50 \mathrm{~mL})$. The organic layer was dried over anhydrous $\mathrm{Na}_{2} \mathrm{SO}_{4}$, filtered and concentrated at $40{ }^{\circ} \mathrm{C}$ without further purification. $R_{\mathrm{f}}=0.62$ (ethyl acetate/petroleum ether, 1:6); m.p. $38-40{ }^{\circ} \mathrm{C} ;{ }^{1} \mathrm{H}$ NMR $\left(400 \mathrm{MHz}, \mathrm{CDCl}_{3}, \mathrm{TMS}\right): \delta=8.04(\mathrm{~d}, J=$ $8.4 \mathrm{~Hz}, 1 \mathrm{H}), 7.98(\mathrm{~d}, J=8.4 \mathrm{~Hz}, 1 \mathrm{H}), 7.36(\mathrm{~m}, 2 \mathrm{H}), 7.31(\mathrm{~d}, J=8.5 \mathrm{~Hz}, 1 \mathrm{H}), 2.75\left(\mathrm{~s}, 3 \mathrm{H}, \mathrm{CH}_{3}\right) ;{ }^{13} \mathrm{C}$ NMR (100 MHz, $\left.\mathrm{CDCl}_{3}, \mathrm{TMS}\right): \delta=158.4,145.4,137.4,135.2,130.3,127.0,122.9,122.1,115.2$, 25.0; IR (KBr): vbar $=2150,2124,1601,1500,1297 \mathrm{~cm}^{-1}$; TOFMS (ESI) calcd for $\mathrm{C}_{10} \mathrm{H}_{9} \mathrm{~N}_{4}$ : $185.0827\left([\mathrm{M}+\mathrm{H}]^{+}\right)$, found 185.0826 .

6-Azidoquinoline-2-carbaldehyde (4). 6-azido-2-methylquinoline (100 mg, $0.54 \mathrm{mmol}$ ) was dissolved in $2 \mathrm{~mL}$ dioxane, and then added to $3 \mathrm{~mL}$ dioxane suspension with $\mathrm{SeO}_{2}(72 \mathrm{mg}, 1.20$ mmol). The mixture reacted at $60^{\circ} \mathrm{C}$ for $6 \mathrm{~h}$ under $\mathrm{N}_{2}$ atmosphere. After the reaction was completed, dioxane were removed in vacuo, and purification by column chromatography (ethyl acetate/petroleum ether, 1:30) gave 6-azidoquinoline-2-carbaldehyde (75 $\mathrm{mg}, 70 \%$ ) as a yellow solid. $R_{\mathrm{f}}=0.60$ (ethyl acetate/petroleum ether, 1/6); m.p. $131-133{ }^{\circ} \mathrm{C} ;{ }^{1} \mathrm{H} \mathrm{NMR}\left(400 \mathrm{MHz}, \mathrm{CDCl}_{3}\right.$, TMS): $\delta=10.20(\mathrm{~d}, J=0.8 \mathrm{~Hz}, 1 \mathrm{H}, \mathrm{CHO}), 8.23(\mathrm{~m}, 2 \mathrm{H}), 8.04(\mathrm{~d}, J=8.5 \mathrm{~Hz}, 1 \mathrm{H}), 7.49(\mathrm{~m}, 2 \mathrm{H})$; ${ }^{13} \mathrm{C}$ NMR (100 MHz, $\left.\mathrm{CDCl}_{3}, \mathrm{TMS}\right): \delta=193.3(\mathrm{CHO}), 152.0,145.6,141.1,136.1,132.5,130.8$, 
123.4, 118.4, 115.2; IR (KBr): vbar $=2119,1707,1618,1467,1284 \mathrm{~cm}^{-1}$; TOFMS (ESI) calcd for $\mathrm{C}_{10} \mathrm{H}_{7} \mathrm{~N}_{4} \mathrm{O}: 199.0620\left([\mathrm{M}+\mathrm{H}]^{+}\right)$, found 199.0620.

Diethyl 2-((6-azidoquinolin-2-yl)methylene)malonate $\quad\left(\mathbf{Q M E}-\mathbf{N}_{3}\right) . \quad 6$-azidoquinoline-2carbaldehyde (100 mg, $0.5 \mathrm{mmol})$ was added to the solution of malonic acid diethyl ester (105 $\mathrm{mg}$, $0.5 \mathrm{mmol})$ in ethanol $(5 \mathrm{~mL})$ and stirred at $50{ }^{\circ} \mathrm{C}$ for $4 \mathrm{~h}$ in the presence of piperidine of catalytic amount $(10 \mathrm{~mol} \%)$ under $\mathrm{N}_{2}$ atmosphere. Solvent in the reaction mixture was removed and the crude product was subjected to column chromatography (ethyl acetate/petroleum ether, 1:5) to afford diethyl 2-((6-azidoquinolin-2-yl)methylene)malonate $(85 \mathrm{mg}, 49 \%)$ as a white solid; $\mathrm{R}_{\mathrm{f}}=$ 0.30 (ethyl acetate/petroleum ether, 1:4); m.p. 96-97 ${ }^{\circ} \mathrm{C} ;{ }^{1} \mathrm{H}$ NMR (400 MHz, $\mathrm{CDCl}_{3}, \mathrm{TMS}$ ): $\delta=$ 8.09 (d, $J=8.5 \mathrm{~Hz}, 1 \mathrm{H}), 7.97$ (d, $J=10.2 \mathrm{~Hz}, 1 \mathrm{H}), 7.79$ (s, 1H), 7.50 (d, $J=8.4 \mathrm{~Hz}, 1 \mathrm{H}), 7.38$ (m, 2H), 4.48 (q, $J=7.2 \mathrm{~Hz}, 2 \mathrm{H}, \mathrm{CH}_{2} \mathrm{CH}_{3}$ ), 4.35 (q, $J=7.2 \mathrm{~Hz}, 2 \mathrm{H}, \mathrm{CH}_{2} \mathrm{CH}_{3}$ ), 1.37 (t, $J=7.1 \mathrm{~Hz}, 3 \mathrm{H}$, $\left.\mathrm{CH}_{2} \mathrm{CH}_{3}\right), 1.36\left(\mathrm{t}, J=7.1 \mathrm{~Hz}, 3 \mathrm{H}, \mathrm{CH}_{2} \mathrm{CH}_{3}\right) ;{ }^{13} \mathrm{C} \mathrm{NMR}\left(100 \mathrm{MHz}, \mathrm{CDCl}_{3}, \mathrm{TMS}\right): \delta=166.4(\mathrm{COO})$, 163.9 (COO), 150.5, 145.6, 139.5, 139.0, 135.6, 131.9, 130.0, 128.6, 123.8, 123.2, 115.0, 61.9, 61.4, 14.2, 14.1; IR (KBr): vbar $=2983,2111,1612,1497,1266,1221 \mathrm{~cm}^{-1}$; TOFMS (ESI) calcd for $\mathrm{C}_{17} \mathrm{H}_{16} \mathrm{~N}_{4} \mathrm{O}_{4} \mathrm{Na}: 363.1069\left([\mathrm{M}+\mathrm{Na}]^{+}\right)$, found 363.1068 .

Tert-butyl (2-methylquinolin-6-yl)carbamate (6). ${ }^{2}{ }^{1} \mathrm{H}$ NMR (400 MHz, $\left.\mathrm{CDCl}_{3}, \mathrm{TMS}\right): \delta=8.06$ (s, 1H), $7.99(\mathrm{~d}, J=8.3 \mathrm{~Hz}, 1 \mathrm{H}), 7.95(\mathrm{~d}, J=8.6 \mathrm{~Hz}, 1 \mathrm{H}), 7.42\left(\mathrm{dd}, J_{1}=9.0, J_{2}=2.4 \mathrm{~Hz}, 1 \mathrm{H}\right), 7.25$ $(\mathrm{d}, J=7.6 \mathrm{~Hz}, 1 \mathrm{H}), 6.68(\mathrm{br}, 1 \mathrm{H}, \mathrm{NH}), 2.72\left(\mathrm{~s}, 3 \mathrm{H}, \mathrm{CH}_{3}\right), 1.55\left(\mathrm{~s}, 9 \mathrm{H}, \mathrm{C}\left(\mathrm{CH}_{3}\right)_{3}\right)$.

Tert-butyl (2-formylquinolin-6-yl)carbamate (7). ${ }^{2}{ }^{1} \mathrm{H}$ NMR (400 MHz, $\left.\mathrm{CDCl}_{3}, \mathrm{TMS}\right): \delta=10.19$ $(\mathrm{d}, J=0.8 \mathrm{~Hz}, 1 \mathrm{H}, \mathrm{CHO}), 8.19(\mathrm{~m}, 3 \mathrm{H}), 7.99(\mathrm{~d}, J=8.5 \mathrm{~Hz}, 1 \mathrm{H}), 7.58\left(\mathrm{dd}, J_{l}=9.1, J_{2}=2.5 \mathrm{~Hz}\right.$, 1H), 6.86 (br, $1 \mathrm{H}, \mathrm{NH}), 1.57\left(\mathrm{~s}, 9 \mathrm{H}, \mathrm{C}\left(\mathrm{CH}_{3}\right)_{3}\right)$.

Diethyl 2-((6-((tert-butoxycarbonyl)amino)quinolin-2-yl)methylene) malonate (8). Tert-butyl (2-formylquinolin-6-yl)carbamate $(0.16 \mathrm{~g}, 0.6 \mathrm{mmol})$ was added to the solution of malonic acid diethyl ester ( $94 \mathrm{mg}, 0.6 \mathrm{mmol})$ in ethanol $(4 \mathrm{~mL})$ and stirred at $50{ }^{\circ} \mathrm{C}$ for $4 \mathrm{~h}$ in the presence of piperidine of catalytic amount $(10 \mathrm{~mol} \%)$ under $\mathrm{N}_{2}$ atmosphere. Solvent in the reaction mixture was removed and the crude product was subjected to column chromatography (ethyl acetate/petroleum ether, 1:5) to afford diethyl 2-((6-((tert-butoxycarbonyl)amino) quinolin-2-yl)methylene) malonate $(0.19 \mathrm{mg}, 77 \%)$ as a white solid. $R_{\mathrm{f}}=0.30$ (ethyl acetate/petroleum ether, $1 / 2$ ); m.p.141-143 ${ }^{\circ} \mathrm{C} ;{ }^{1} \mathrm{H}$ NMR (400 MHz, $\left.\mathrm{CDCl}_{3}, \mathrm{TMS}\right): \delta=8.07(\mathrm{~m}, 2 \mathrm{H}), 7.87(\mathrm{~d}, J=9.0 \mathrm{~Hz}, 1 \mathrm{H}), 7.78(\mathrm{~s}, 1 \mathrm{H}), 7.44(\mathrm{dd}$, 
$\left.J_{1}=9.1, J_{2}=2.3 \mathrm{~Hz}, 2 \mathrm{H}\right), 6.76(\mathrm{~s}, 1 \mathrm{H}, \mathrm{NH}), 4.49\left(\mathrm{q}, J=7.2 \mathrm{~Hz}, 2 \mathrm{H}, \mathrm{CH}_{2} \mathrm{CH}_{3}\right), 4.34(\mathrm{q}, J=7.2 \mathrm{~Hz}$, $\left.2 \mathrm{H}, \mathrm{CH}_{2} \mathrm{CH}_{3}\right), 1.56\left(\mathrm{~s}, 9 \mathrm{H}, \mathrm{C}\left(\mathrm{CH}_{3}\right)_{3}\right), 1.35\left(\mathrm{~m}, 6 \mathrm{H}, \mathrm{CH}_{2} \mathrm{CH}_{3}\right) ;{ }^{13} \mathrm{C} \mathrm{NMR}\left(100 \mathrm{MHz}, \mathrm{CDCl}_{3}, \mathrm{TMS}\right): \delta$ $=166.8$ (COO), 164.0 (COO), 152.6, 149.3, 144.6, 139.6, 137.4, 136.0, 130.6, 129.1, 128.8, 123.4, 123.2, 113.0, 81.1, 61.8, 61.4, 28.3, 14.1; IR (KBr): vbar = $3318(\mathrm{NH}), 2980,1719,1622,1493$, $1277 \mathrm{~cm}^{-1}$; TOFMS (ESI) calcd for $\mathrm{C}_{22} \mathrm{H}_{27} \mathrm{~N}_{2} \mathrm{O}_{6}: 415.1869\left([\mathrm{M}+\mathrm{H}]^{+}\right)$, found 415.1870 .

Diethyl 2-((6-aminoquinolin-2-yl)methylene)malonate $\quad\left(\mathbf{Q M E}-\mathbf{N H}_{2}\right) . \quad$ Diethyl 2-((6-((tertbutoxycarbonyl)amino)quinolin-2-yl)methylene)malonate (30 $\mathrm{mg}, 0.072 \mathrm{mmol}$ ) was dissoled in 2 $\mathrm{mL}$ of the mixed solution (trifluoroacetic acid/dichloromethane, 1:1). The mixture was stirred at room temperature overnight. After the reaction was completed, the solution was quenched by $10 \%$ solution of sodium bicarbonate, extracted with dichloromethane. The crude product was subjected to column chromatography (ethyl acetate/petroleum ether, 1:2) to afford diethyl 2-((6-aminoquinolin-2-yl)methylene)malonate $(14 \mathrm{mg}, 62 \%)$ as a yellow solid; $R_{\mathrm{f}}=0.35$ (ethyl acetate/petroleum ether, 1:1); m.p. $126-128{ }^{\circ} \mathrm{C} ;{ }^{1} \mathrm{H}$ NMR $\left(400 \mathrm{MHz}, \mathrm{CDCl}_{3}\right) \delta(\mathrm{ppm}): 7.87(\mathrm{~d}, J=$ $8.4 \mathrm{~Hz}, 1 \mathrm{H}), 7.78(\mathrm{~m}, 2 \mathrm{H}), 7.37(\mathrm{~d}, J=8.4 \mathrm{~Hz}, 1 \mathrm{H}), 7.14\left(\mathrm{dd}, J_{l}=2.4, J_{2}=9.0 \mathrm{~Hz}, 1 \mathrm{H}\right), 6.85(\mathrm{~d}, J=$ 2. $3 \mathrm{~Hz}, 1 \mathrm{H}), 4.49\left(\mathrm{q}, J=7.2 \mathrm{~Hz}, 2 \mathrm{H}, \mathrm{CH}_{2} \mathrm{CH}_{3}\right), 4.33\left(\mathrm{q}, J=7.1 \mathrm{~Hz}, 2 \mathrm{H}, \mathrm{CH}_{2} \mathrm{CH}_{3}\right), 1.36(\mathrm{~m}, 6 \mathrm{H}$, $\left.\mathrm{CH}_{2} \mathrm{CH}_{3}\right) ;{ }^{13} \mathrm{C}$ NMR (100 MHz, $\mathrm{CDCl}_{3}$, TMS): $\delta=167.0$ (COO), 164.2 (COO), 147.1, 145.9, 143.1, $140.0,133.9,131.3,129.9,128.0,123.5,122.3,106.6,61.6,61.3,14.2$; IR (KBr): vbar $=3460$ $(\mathrm{NH}), 3358(\mathrm{NH}), 2978,1719,1685,1614,1481,1281 \mathrm{~cm}^{-1}$; TOFMS (ESI) calcd for $\mathrm{C}_{17} \mathrm{H}_{18} \mathrm{~N}_{2} \mathrm{O}_{4} \mathrm{Na}$ : $337.1164\left([\mathrm{M}+\mathrm{Na}]^{+}\right)$, found 337.1167.

Diethyl 2-((6-aminoquinolin-2-yl)(propylthio)methyl)malonate (QME-NH2-SPr). QME-NH (35 mg, $0.22 \mathrm{mmol}$ ) was dissolved in the mixed solution of ethanol/water (1:2), 1-propanethoil (60 $\mu \mathrm{L}$, large excess) was added into the solution and stirred at room temperature for overnight. The reaction mixture was extracted with ethyl acetate $(3 \times 15 \mathrm{~mL})$. The combined organic layer was dried over $\mathrm{MgSO}_{4}$, filtered and evaporated under reduced pressure. The crude product was purified by silica gel column chromatography (petroleum ether/ethyl acetate, 10:1) affording QME-NH2-SR as a yellow liquid $(27 \mathrm{mg}, 63 \%) . R_{\mathrm{f}}=0.45$ (ethyl acetate/petroleum ether, 1:2); ${ }^{1} \mathrm{H}$ NMR (400 MHz, $\left.\mathrm{CDCl}_{3}, \mathrm{TMS}\right): \delta=7.84(\mathrm{~d}, J=8.6 \mathrm{~Hz}, 1 \mathrm{H}$, quinoline-H), 7.77 (d, $J=8.9 \mathrm{~Hz}, 1 \mathrm{H}$, quinoline-H), $7.43\left(\mathrm{~d}, J=8.6 \mathrm{~Hz}, 1 \mathrm{H}\right.$, quinoline-H), $7.09\left(\mathrm{dd}, J_{l}=2.6, J_{2}=8.9 \mathrm{~Hz}, 1 \mathrm{H}\right.$, quinoline-H), $6.86(\mathrm{~d}, J=$ $2.6 \mathrm{~Hz}, 1 \mathrm{H}$, quinoline-H), 4.69 (d, $J=11.5 \mathrm{~Hz}, 1 \mathrm{H}, \mathrm{CHS}), 4.62$ (d, $J=11.5 \mathrm{~Hz}, 1 \mathrm{H}, \mathrm{CHCO}), 4.30$ 
(m, 2H, $\left.\mathrm{OCH}_{2} \mathrm{CH}_{3}\right), 4.01$ (q, $\left.J=7.1 \mathrm{~Hz}, 2 \mathrm{H}, \mathrm{OCH}_{2} \mathrm{CH}_{3}\right), 2.46-2.25\left(\mathrm{~m}, 2 \times 1 \mathrm{H}, \mathrm{SCH}_{2}\right), 1.36(\mathrm{~m}$, $(2+3) \mathrm{H}, \mathrm{CH}_{3} \mathrm{CH}_{2} \mathrm{CH}_{2} \mathrm{~S}$ and $\left.\mathrm{OCH}_{2} \mathrm{CH}_{3}\right), 1.04\left(\mathrm{t}, J=7.1 \mathrm{~Hz}, 3 \mathrm{H}, \mathrm{OCH}_{2} \mathrm{CH}_{3}\right), 0.80(\mathrm{t}, J=7.3 \mathrm{~Hz}, 3 \mathrm{H}$, $\left.\mathrm{CH}_{2} \mathrm{CH}_{2} \mathrm{CH}_{3}\right) ;{ }^{13} \mathrm{C} \mathrm{NMR}\left(100 \mathrm{MHz}, \mathrm{CDCl}_{3}, \mathrm{TMS}\right): \delta=168.1$ (COO), 167.9 (COO), 155.4, 144.6, $141.7,134.5,130.3,128.4,121.7,121.1,107.6,61.7,61.3,55.9,48.4,31.0,22.7,14.2,13.9,13.4$; IR $(\mathrm{KBr}): \operatorname{vbar}=3468(\mathrm{NH}), 3378(\mathrm{NH}), 2964,1748,1731,1631,1506,1369,1242 \mathrm{~cm}^{-1}$; TOFMS (ESI) calcd for $\mathrm{C}_{20} \mathrm{H}_{27} \mathrm{~N}_{2} \mathrm{O}_{4} \mathrm{~S}: 391.1692\left([\mathrm{M}+\mathrm{H}]^{+}\right)$, found 391.1699.

\section{References}

(1) Saravanan, M.; Satyanarayana, B.; Reddy, P. P. New and Practical Synthesis of Montelukast Sodium, an Antiasthmatic Drug. Syn. Commun. 2013, 43, 2050-2056.

(2) Butler, S. J. Ratiometric Detection of Adenosine Triphosphate (ATP) in Water and Real-Time Monitoring of Apyrase Activity with a Tripodal Zinc Complex. Chem.-Eur. J. 2014, 20, 15768-15774. 
VIII. Copies of NMR spectra of related compounds

Copies of NMR spectra of compound 3
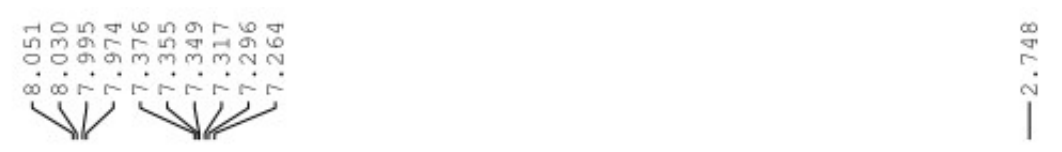

$\circ$
0
1
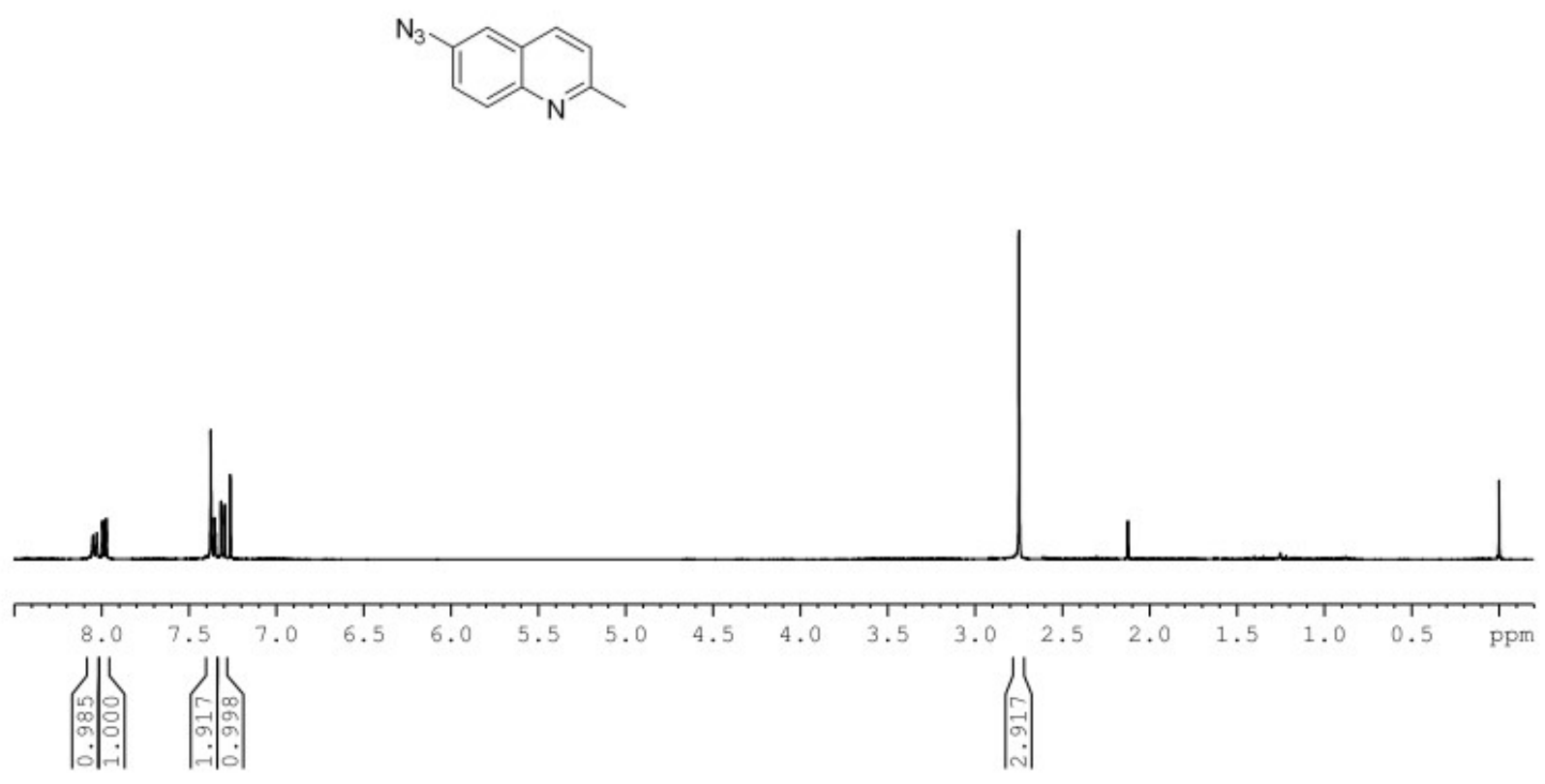

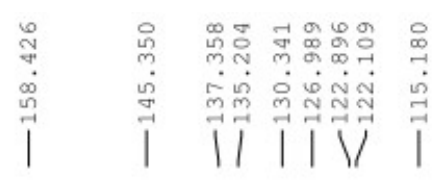

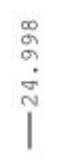
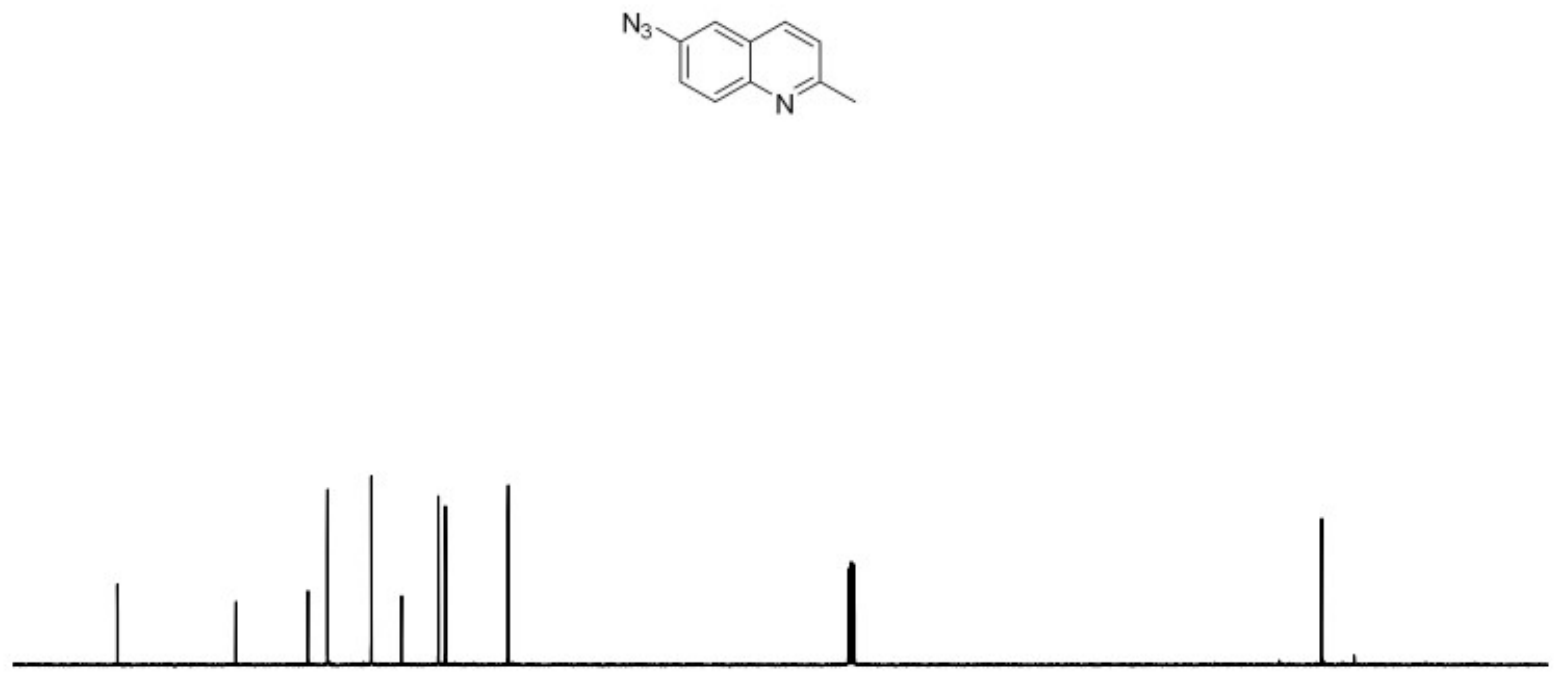

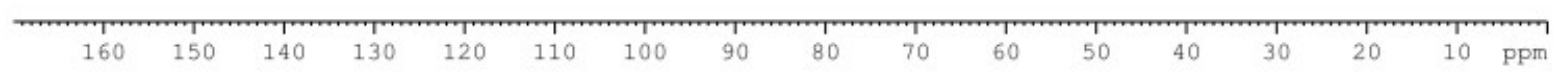

$\mathrm{S} 12$ 
Copies of NMR spectra of compound 4
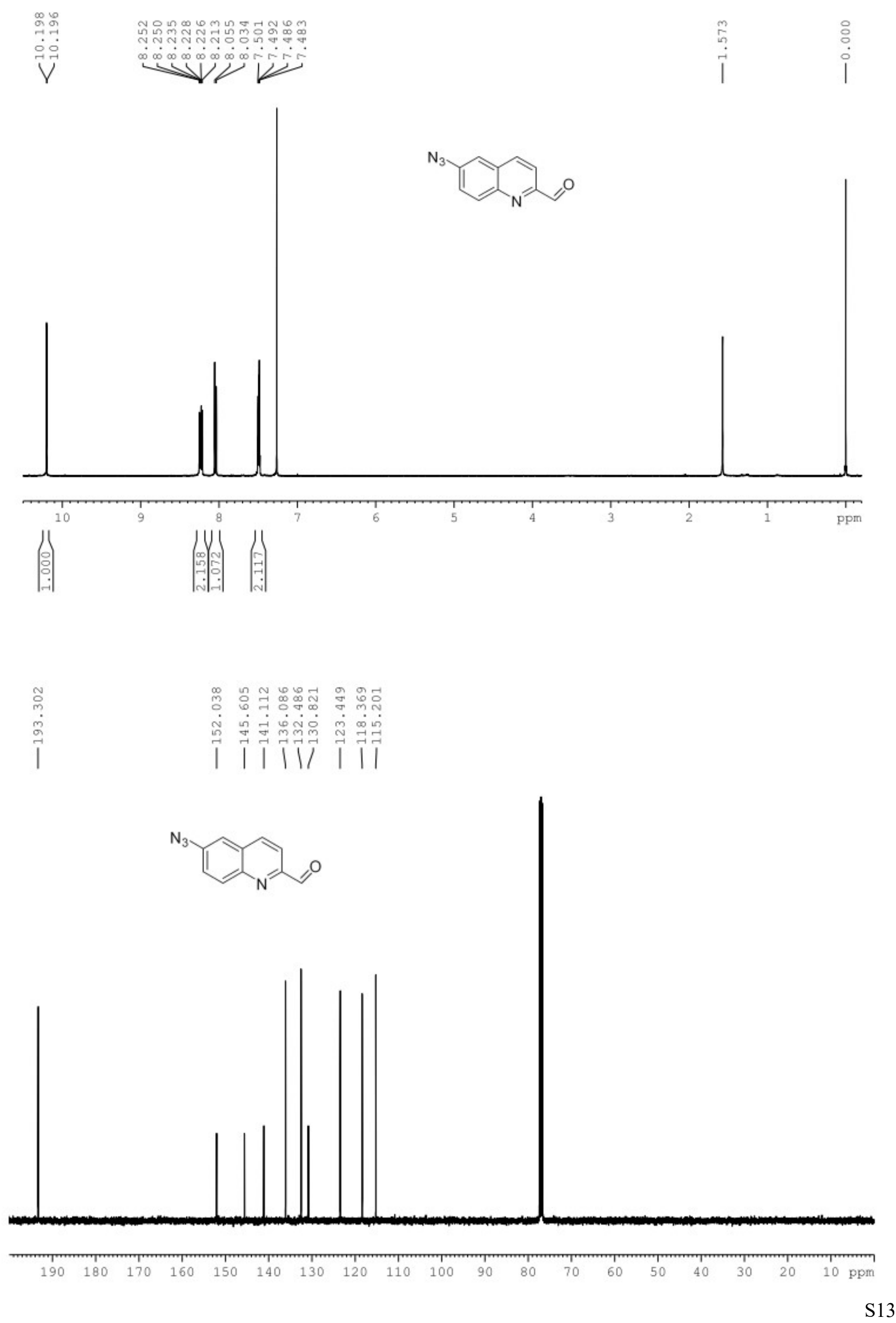
Copies of NMR spectra of QME-N 3

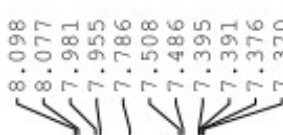

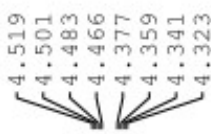

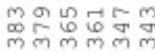

viv
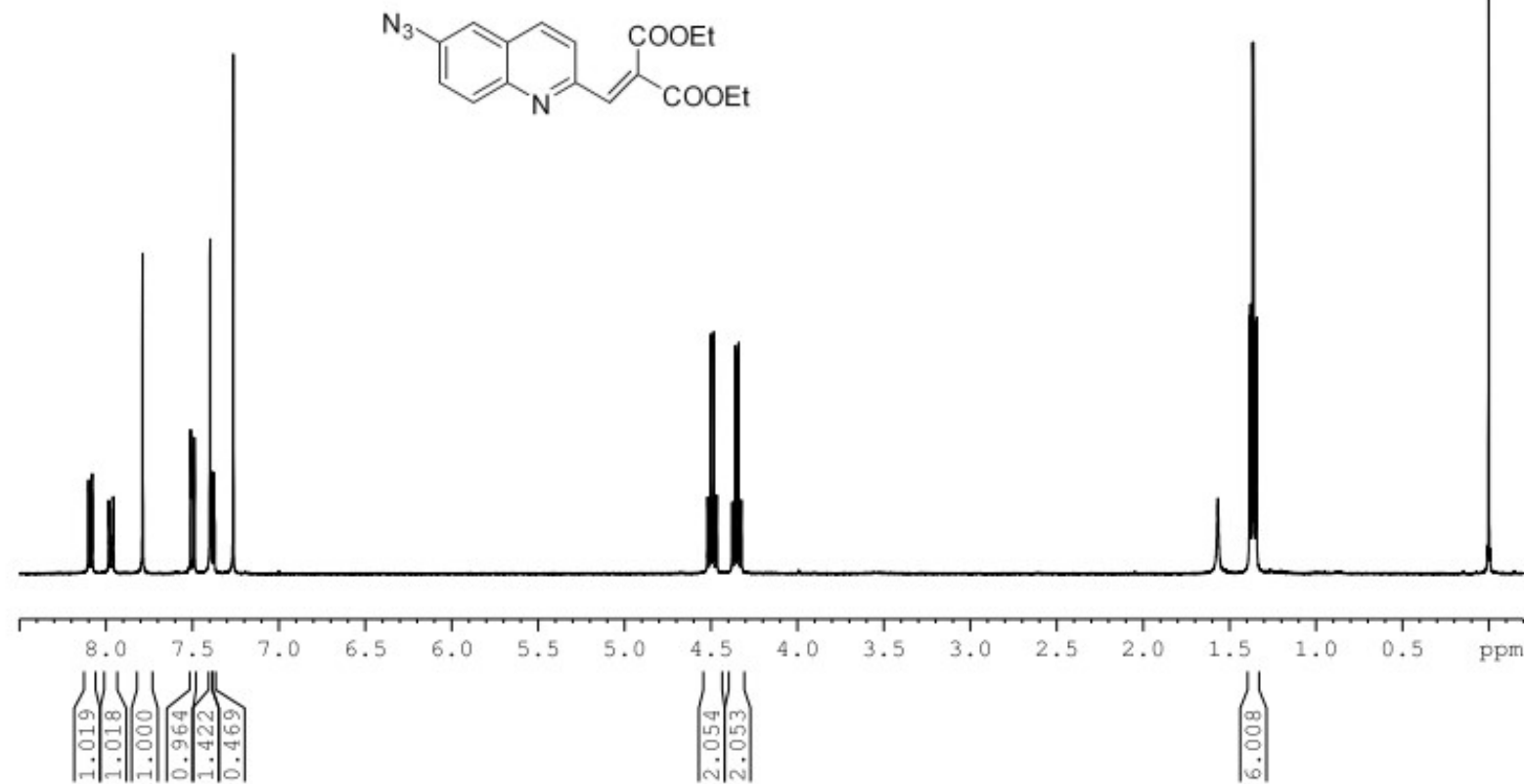

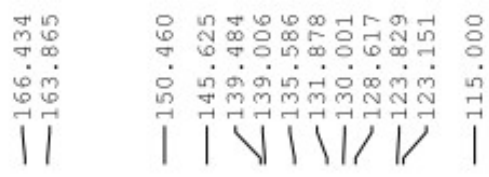
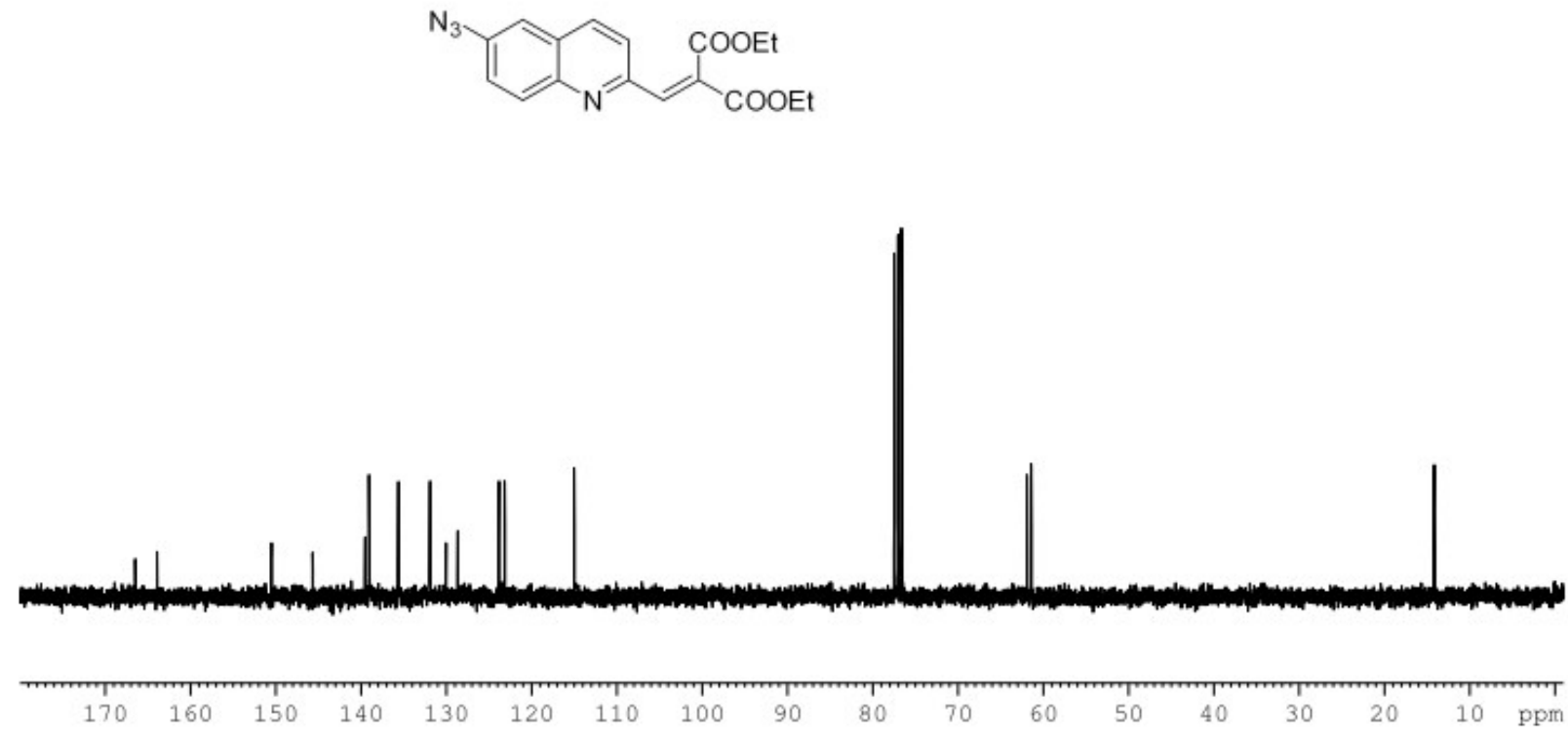
Copies of NMR spectra of compound 7

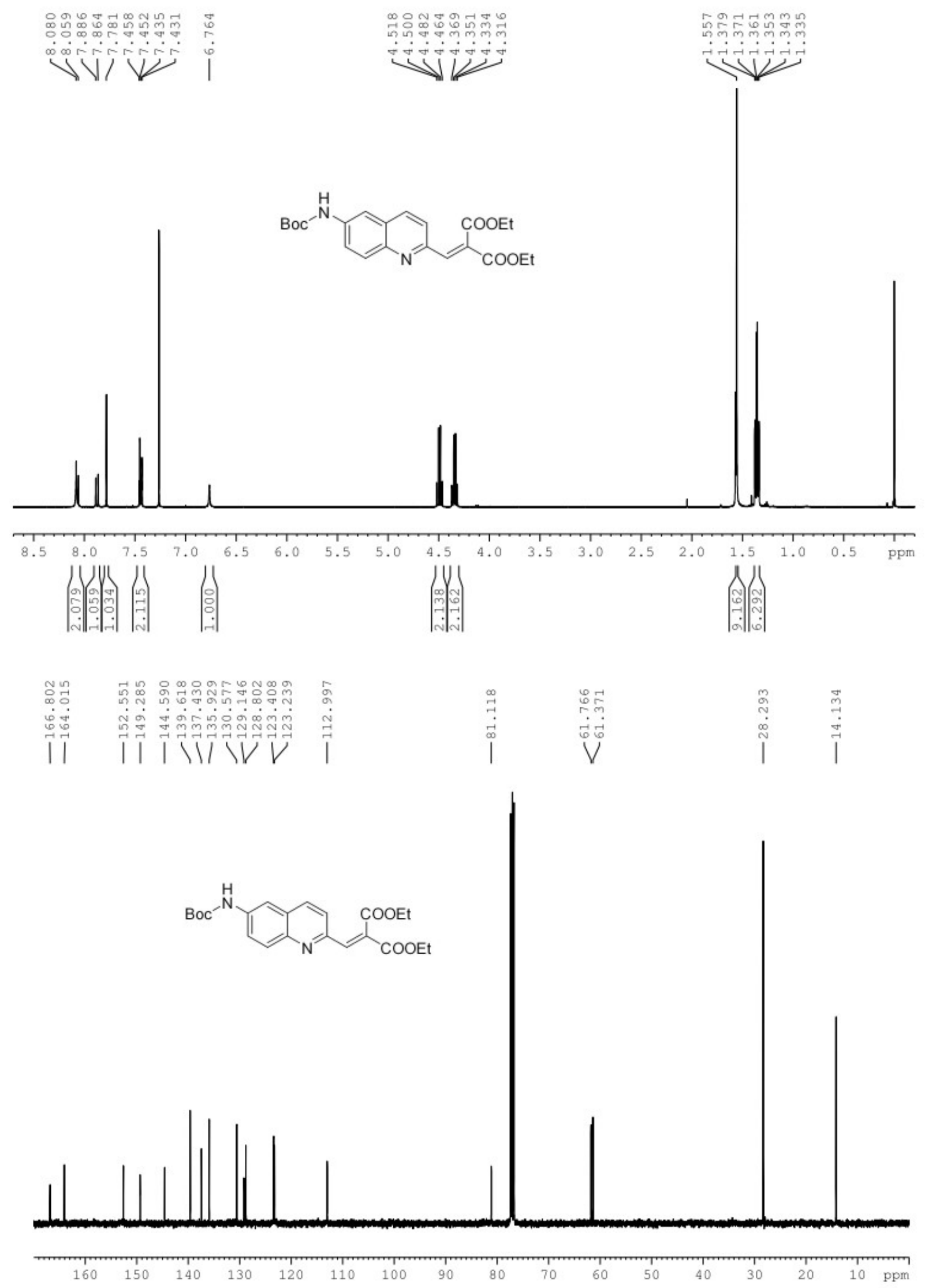


Copies of NMR spectra of QME-NH
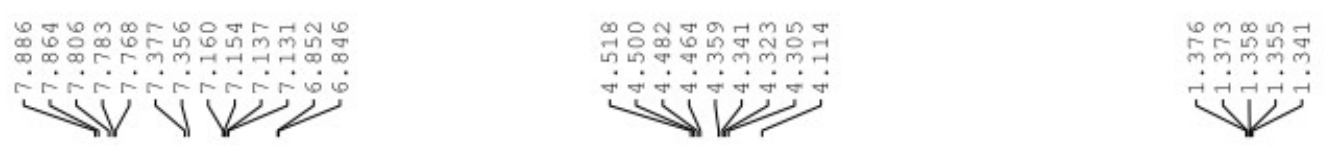

COOEt
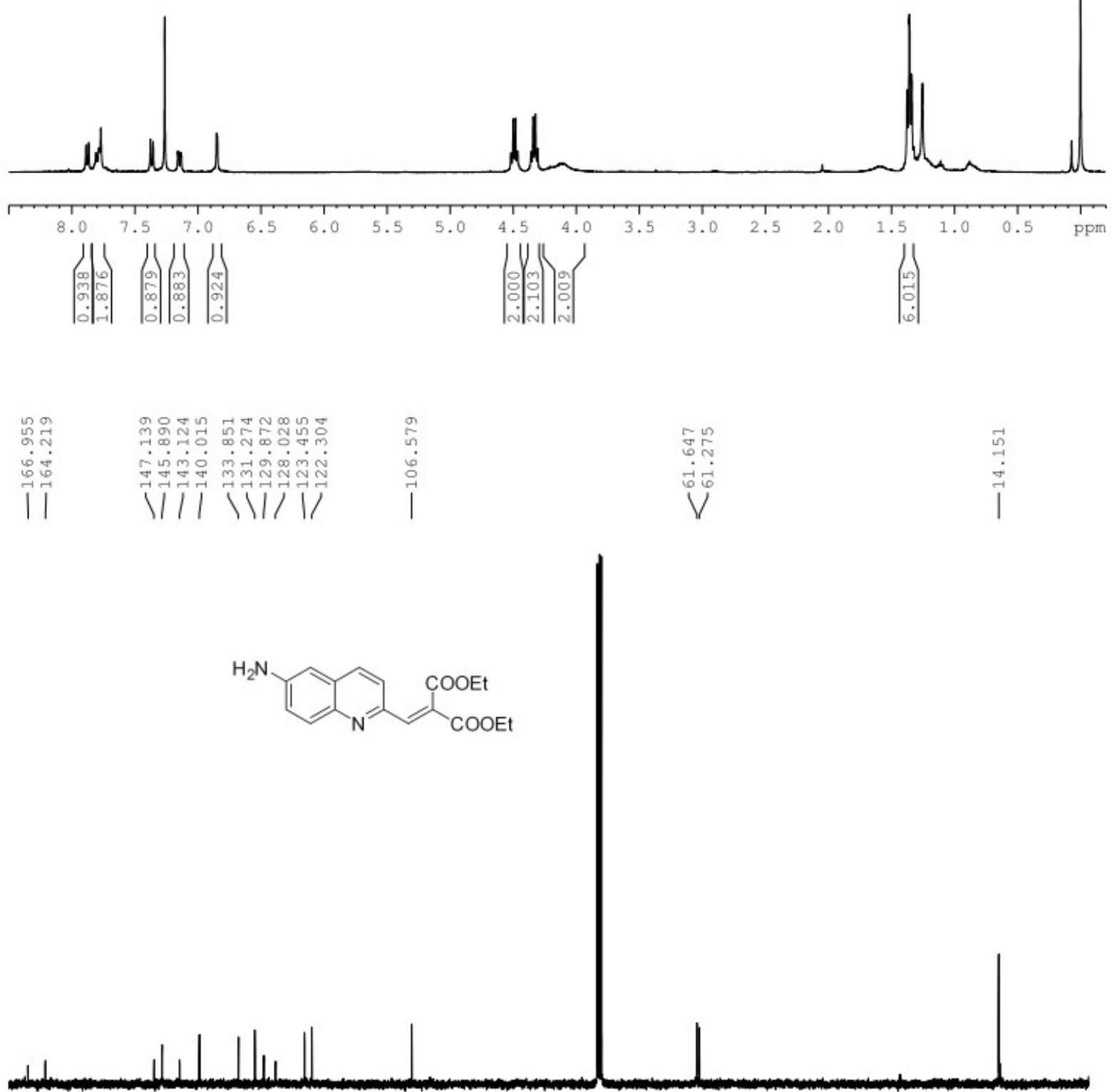

160
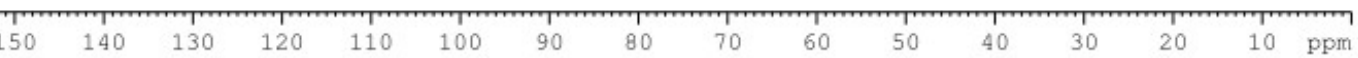
Copies of NMR spectra of QME-NH $\mathrm{N}_{2}-\mathrm{SPr}$

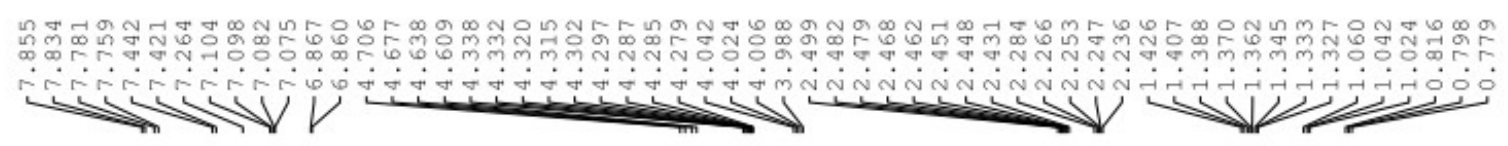<smiles>CCCSC(c1ccc2cc(N)ccc2n1)C(C(=O)OCC)C(=O)OCC</smiles>

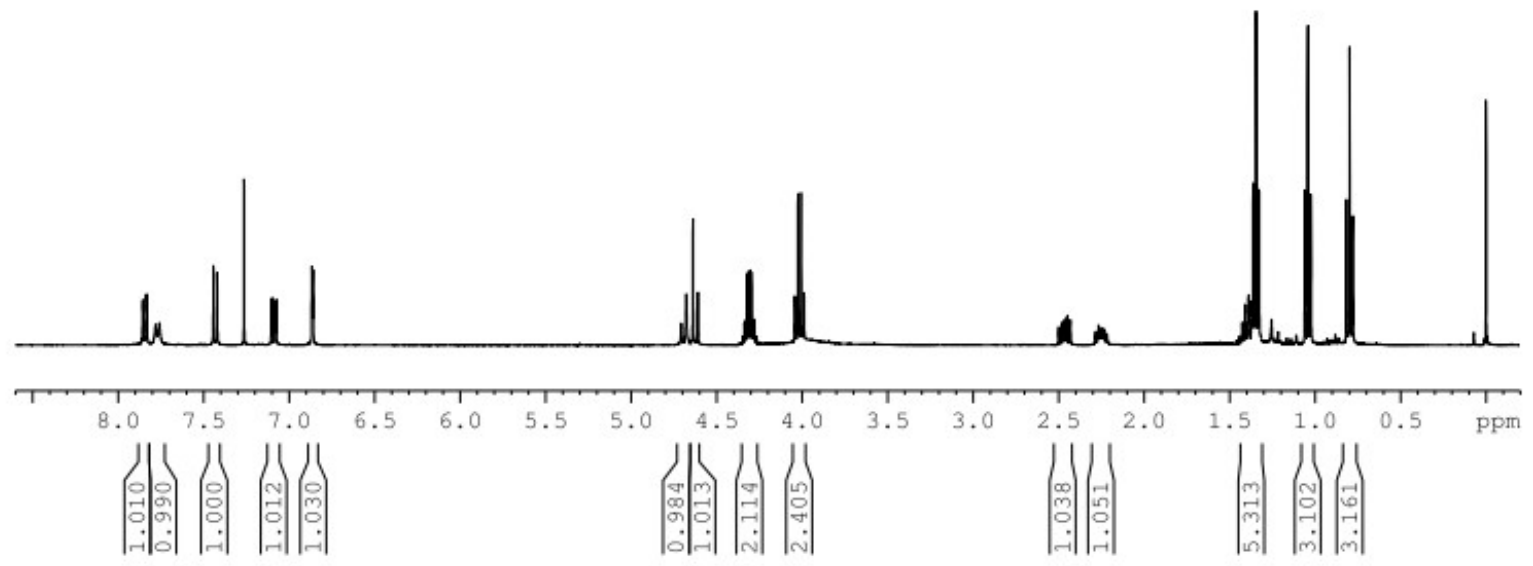

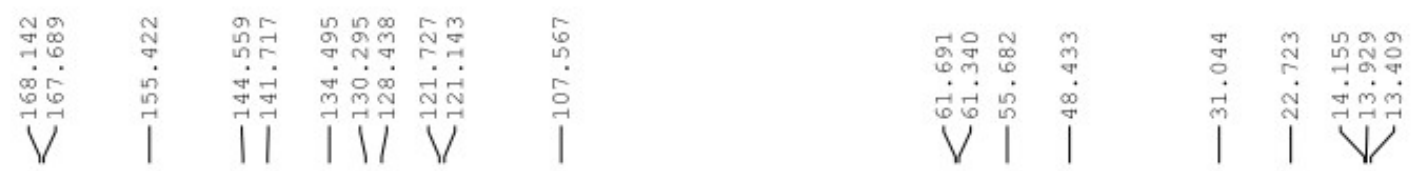<smiles>CCCSC(c1ccc2cc(N)ccc2n1)C(C(=O)OCC)C(=O)OCC</smiles>

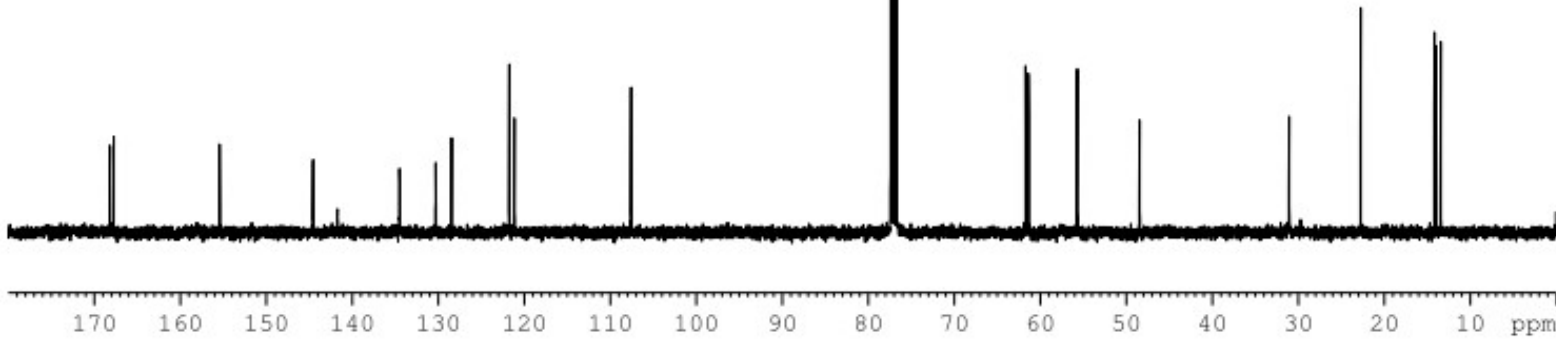

